\title{
Self-controlled Sequencing Suggests that Somatic Mutations of Signaling, Transcription and Tumor Suppression Genes are a Precondition for AML Transformation in MDS
}

\section{Feng Xu}

Shanghai 6th Peoples Hospital Affiliated to Shanghai Jiaotong University

Ling-Yun Wu

Shanghai 6th Peoples Hospital Affiliated to Shanghai Jiaotong University

Juan Guo

Shanghai 6th Peoples Hospital Affiliated to Shanghai Jiaotong University

Qi He

Shanghai 6th Peoples Hospital Affiliated to Shanghai Jiaotong University

\section{Zheng Zhang}

Shanghai 6th Peoples Hospital Affiliated to Shanghai Jiaotong University

Xiao Li (D lixiao3326@163.com)

Shanghai 6th Peoples Hospital Affiliated to Shanghai Jiaotong University https://orcid.org/0000-0002-7024-4444

\section{Research Article}

Keywords: myelodysplastic syndromes, sequencing, somatic mutations, AML transformation

Posted Date: January 11th, 2022

DOI: https://doi.org/10.21203/rs.3.rs-1142924/v1

License: () (1) This work is licensed under a Creative Commons Attribution 4.0 International License. Read Full License 


\section{Abstract \\ Background}

The transformation biology of secondary AML from MDS is still not fully understood. Here, we performed a large cohort of paired self-controlled sequences including target, whole-exome and single cell sequencing to search AML transformation-related mutations (TRMs).

\section{Methods}

39 target genes from paired samples from 72 patients with MDS who had undergone AML transformation were analyzed by next generation target sequencing. Whole exome and single-cell RNA sequencing were used to verify the dynamics of transformation.

\section{Results}

The target sequencing results showed that sixty-four out of the 72 (88.9\%) patients presented presumptive TRMs involving activated signaling, transcription factors, or tumor suppressors. Of the 64 patients, most of TRMs (62.5\%, 40 cases) emerged at the leukemia transformation point. All three of the remaining eight patients analyzed by paired whole exome sequencing showed TRMs which are not included in the reference targets. No patient with MDS developed into AML only by acquiring mutations involved in epigenetic modulation or RNA splicing. Single-cell sequencing in one pair sample indicated that the activated cell signaling route was related to TRMs which take place prior to phenotypic development. Of note, target sequencing defined TRMs were limited to a small set of seven genes (in the order: NRAS/KRAS, CEBPA, TP53, FLT3, CBL, PTPN11 and RUNX1, accounted for nearly $90.0 \%$ of the TRMs).

\section{Conclusions}

Somatic mutations involving in signaling, transcription factors, or tumor suppressors appeared to be a precondition for AML transformation from MDS. The TRMs may be considered as new therapy targets.

\section{Introduction}

In most cases, de novo acute myeloid leukemia (AML) shows rapid onset without an obvious pre-AML period [1]. Patients have been reported to show special genetic abnormalities in different subsets [2-4]. Somatic mutations involving CEBPa, FLT3, NPM1, and c-Kit have been used to assess prognosis [5]. Based on these tumor-derived biological characteristics, target-specific and immunological methods have been developed to treat AML [5]. Unlike de novo AML, secondary AML (sAML), transformed from myelodysplastic syndromes (MDS), shows unique genetic features [6]. First, there is often an obvious pre-AML stage, where somatic gene mutations result in initial events (clonal hematopoiesis) and/or driver events (development of MDS phenotypes), largely involving epigenetic regulation, RNA splicing, fewer others [7, 8]. Second, despite preliminary findings on the roles of late-stage gene mutations involving various signaling pathways or transcription during SAML development $[9,10]$, including our primary consideration on sAML-related mutations [11], the transformation biology of sAML is still not fully understood.

There are still many unanswered questions in SAML transformation biology. Could early or late somatic mutations solely involved in epigenetic regulation or RNA splicing also induce SAML? Which mutations are the key factors that induce the transformation process? When do the transformation-related mutations emerge? Are they pre-existing at MDS diagnosis, or do they emerge when the transformation starts? Answering of these questions will help us to find new insight of SAML pathogenesis, and then explore some new target therapy strategies. Logically, only systematized finding, but not some sporadic reports could answer these doubts. A systematized finding in this assay means the data should come from a big size sample, which could offer some frequency materials to focus our attention to some high occurrence rate mutations related to sAML transformations. In addition, a systematized finding should come from a paired self-controlled data, which would clearly tell us what mutations initiate MDS development, and what other mutations trigger off sAML transformation. Finally, the objects of observation in this study are focused on those mutated genes related to switch the sAML transformation, rather than an overall MDS clonal evolution [9]. In general, the functions of those mutations occur during early/middle stage of MDS (such as those MDS special gene mutations involving epigenetic regulation and RNA splicing) is to initiate clonal hematopoiesis and keep the phenotypes of MDS themselves. In theory, only those late events referring to signaling, transcription or tumor suppressor could start the sAML transformation process [7]. So the purpose of this study is to observe what happens on gene mutation when a MDS develops into sAML.

Paired self-controlled samples were acquired at MDS diagnosis and immediately after AML transformation. Sequencing for 39 target genes in all samples was followed by whole exome sequencing in the few patients whose target sequencing did not identify novel mutations. An additional sample pair was subjected to single-cell RNA transcription sequencing. Using these techniques, we obtained some useful data that informs the biology of sAML transformation and suggests some novel strategies to block leukemic transformation of MDS.

\section{Patients And Methods}

\section{Sample collection}

From January 2004 until October 2020, a total of 1,541 patients were diagnosed with MDS in our department. Over $90 \%$ were followed up by calls or with faceto-face consultations, and over 800 patients consented to provide samples for next generation sequencing. Targeted sequencing was performed using paired 
samples (at the MDS diagnosis and AML transformation points) to detect transformation-related gene mutations (TRMs). Whole exome sequencing was performed when conclusive results for last mutation events were not obtained via targeted sequencing and when adequate residual DNA extracts were available. Single-cell RNA transcription sequencing was used to examine the underlying transformation dynamics. Diagnoses for MDS and sAML were established in strict accordance with the WHO criteria, and the CMML subset were also included in this assay according to the FAB classification [12, 13].Clinical and hematologic data were recorded following informed consent in accordance with the Declaration of Helsinki; this study was approved by hospital review boards of the Shanghai Jiao Tong University Affiliated Sixth People's Hospital.

\section{Genomic DNA preparation, target enrichment, and sequencing}

Genomic DNA (gDNA) was extracted using the DNeasy Blood and Tissue Kit (Qiagen, Germany) according to the manufacturer's protocol. Genomic DNA was sheared using the Covaris ${ }^{\circledR}$ system (Covaris, USA), and the DNA sample was prepared using the Truseq DNA Sample preparation Kit (IIlumina, USA) according to the manufacturer's protocol.

For probe design, both coding and regulatory regions of target genes were included in the custom panel. The regulatory regions comprised promoter regions (defined as $2 \mathrm{~kb}$ upstream of the transcription start site), 5' un-translated region (5'-UTR), and intron-exon boundaries (50 bp). Custom capture oligos were designed using SureDesign website of Agilent Technologies (Agilent, USA). Hybridization reactions were carried out on ABI 2720 Thermal Cycler (Life Technologies, USA) with the following hybridization conditions. Hybridization mixture was incubated for 16 or $24 \mathrm{~h}$ at $65^{\circ} \mathrm{C}$ with a heated lid at $105^{\circ} \mathrm{C}$. After the hybridization reactions, the hybridization mixture was captured and washed with magnetic beads (Invitrogen, USA) and SureSelect target enrichment kit (Agilent, USA). The captured product was enriched with the following cycling conditions, $98^{\circ} \mathrm{C}$ for $30 \mathrm{~s}, 10 \mathrm{cycles}$ of $98^{\circ} \mathrm{C}$ for $10 \mathrm{~s}, 60^{\circ} \mathrm{C}$ for $30 \mathrm{~s}, 72^{\circ} \mathrm{C}$ for $30 \mathrm{~s}$, and $72^{\circ} \mathrm{C}$ for $5 \mathrm{~min}$. Library quality was assessed using an Agilent 2100 Bioanalyzer (Agilent, USA), and multiplexed sequencing was performed on HiSeq 2500 sequencers with $2 \times 150$ paired-end modules (Illumina, USA). Average sequencing depth was $800 \times$. Following 39 sequenced target genes were included: ANKRD11, ASXL1, BCOR, CALR, CBL, CEBPA, DHX9, DNMT3A, ETV6, EZH2, FLT3, GATA2, IDH1, IDH2, ITIH3, JAK2, KIF2OB, KIT, KRAS, MPL, NF1, NPM1, NRAS, PHF6, PTPN11, PTPRD, ROBO1, ROBO2, RUNX1, SETBP1, SF3B1, SRSF2, STAG2, TET2, TP53, U2AF1, UPF3A, WT1, and ZRSR2.

\section{Whole-exome sequencing}

The gDNA library was prepared using a TruSeq DNA Sample Preparation Kit (Illumina, San Diego, CA, US) in accordance with the manufacturer's protocol. Insolution exome enrichment was performed using a TruSeq Exome Enrichment kit (Illumina) according to the manufacturer's instructions. The enriched DNA samples were sequenced via $2 \times 100$ paired-end sequencing using a Hiseq2000 Sequencing System (Illumina). Illumina Sequencing Control v2.8, Illumina OffLine Basecaller v1.8, and Illumina Consensus Assessment of Sequence and Variation v1.8 software were used to produce 100-base pair (bp) sequence reads.

\section{Sequencing data processing, variant calling, and annotation}

Before variant calling, the raw sequence reads were mapped to the reference genome (hg19), duplicate reads were marked and removed to mitigate biases introduced by amplification, and base quality scores were recalibrated using the Genome Analysis Toolkit (GATK). The Ensembl VEP and vcf2maf tools were applied to generate a MAF format for somatic mutation annotation, and ANNOVAR tool was used to annotate frequency information of variations in the population database. The variants were identified as low-frequency functional mutation if they had $<0.1$ frequency in ExAC03 database, $<0.01$ frequency in 1,000 genome database, and $<0.05$ frequency in GeneskyDB database. According to the results, the variant was then extracted as one of the following functional annotations: "Frame_Shift_Del/ins", "In_Frame__Del/Ins", "Missense_Mutation", "Nonsense_Mutation", "Nonstop_Mutation", "Splice_Site" or "Translation_Start_Site".

\section{Single-cell RNA sequencing and bioinformatic data analysis}

Bone marrow mononuclear cells (BMMC) concentrations were measured by hemocytometer and adjusted to $700-2,000$ cells/ $\mu \mathrm{L}$. Single-cell RNA-seq libraries were generated using 10x Genomics Single Cell 3' reagent v2 according to the manufacturer's instructions. Twelve and 14 cycles were used for complementary DNA and index PCR amplifications, respectively. Amplified cDNA and library quality was assessed using Agilent 2100 Bioanalyzer (Agilent, USA). Libraries were pooled and sequenced on HiSeqX Ten platform (Illumina) with $2 \times 150$ paired-end modules generating at least $50 \mathrm{~K}$ mean reads per cell. Raw sequencing data were processed using Cell Ranger version 3.1.0 (10x Genomics) with default parameters. Quality control metrics were used to remove cells with mitochondrial gene percentage more than $20 \%$ or cells with fewer than 200 genes detected. Variably expressed gene selection, dimensionality reduction, and clustering were performed using the Seurat package version 3.1.3. Principal component analysis was performed on significantly variable genes and the first 40 principal components were used for UMAP dimension reduction. Cell type of each cluster was identified using singleR version 1.0.1. For paired samples, differentially expressed genes (fold change $>2$ and Wilcox test $P$ value $<0.05$ ) were identified in each of seven cell types independently, of which the mutation genes PTPN11 and NRAS were used for GO and KEGG enrichment analysis.

\section{Definition of the presumed transformation-related mutation}

When we analyzed the paired data, if pre-existing genes at MDS diagnosis or newly-emerging genes at sAML transformation met following conditions, we considered they are sAML transformation related mutations (TRMs): 1. they must be involved in at least one of the three function pathways namely, active signaling, myeloid transcription or tumor suppression. 2. They emerged after SAML transformation (better weight) or pre-existed at MDS diagnosis (poorer weight). Newly emerged mutations were preferentially considered as the presumed transformation-related mutations. 3 . When $\geq 2$ suspicious mutations coexisted to be defined as transformation-related mutations, the biologically more aggressive one (active signaling > myeloid transcription > tumor suppressor); or with lower VAF (Various allele frequency) among newly emerged mutations (meaning latest emergence) were defined as transformation-related mutations (TRMs). Occasionally, over one mutation could be presumed as TRMs for one patient.

\section{Statistics analysis}


Statistical analyses were conducted using SPSS software version 18.0. Kaplan-Meier analysis was used to evaluate the time to survival and time to progression. All P-values were based on 2-sided tests and P-values less than 0.05 were considered statistically significant.

\section{Results}

\section{Target sequencing}

Paired samples acquired from seventy-two MDS patients before and after transformation to sAML were analyzed using target sequencing. Identified somatic mutations are presented in Table 1. Of course, 72 cases was much less than actually sAML transformed patients number. In other word, because of loss of follow up and rejection of repeat sequencing, only a part of SAML patients were repeatedly sequenced and analyzed here.

1. Transformation-related mutations (TRMs) were identified in sixty-four of the 72 patients (88.9\%), according to the steps described in METHODS.

2. Among these 64, TRMs were detected only at AML transformation in 40 patients (representing $62.5 \%$ of these cases). In the 24 remaining cases, these mutations were detected at the point of MDS diagnosis (Table 1). Patients with TRMs in only their sAML sample trended toward a longer time duration to undergo AML transformation (median 11.5 months vs. 8 months, $P=0.045$ ), but without significant difference in lower risk (<RAEB2/CMML2) cases frequency (77.5\% vs. $75.0 \%, P=0.819)$

3. The total defined TRMs number showed more than 64 , because sometimes over one mutations could be considered as TRMs for one patient, and occasionally the TRMs for one case could be from both signaling and transcriptor. From Table 1, 2 and Figure 1, the following defined TRMs from the 64 patients were present in descending order of frequency. First were active signaling mutations: KRAS/ NRAS in 14 patients; FLT3 in 8 patients; CBL in 7 patients; PTPN11 in 6, PTPRD in 2, KIT and GATA2 in 1 respectively. The next set of mutations comprised myeloid transcription factors: CEBPA in 11 patients; RUNX1 in 5 patients, ETV6 in3, and SETBP1 in 2patients respectively. The next set of mutations comprised tumor suppressor genes: TP53 mutations in 9; WT1 mutations in 3 patients, and NPM1 in 1 patient. The TRMs events seemed to be highly enriched in 7 genes. Figure 1 demonstrated that the involvement style of FLT3 (seven of the 8 cases appeared point mutations) was not the same as that of FLT3-ITD, which is more often observed in de novo AML.

4. In 6 of these 64 paired samples with candidate transformation events, we sequenced an additional sample between MDS diagnosis and AML transformation (Figure 2). Of these, 3 paired samples (UPN1243, 3288, and 4390) showed evidence of the TRMs emerging before phenotype change, i.e., the TRMs event emerged while the disease was still in the MDS stage, with sAML transformation occurring soon thereafter.

5. Analysis of changing mutation profiles and VAF, as well as the logical relations between them, revealed differing patterns of evolution from MDS to sAML. Of the 64 patients with candidate mutation events, most (29 cases) demonstrated AML-transformation-related progression by linear evolution from the founding clone (Figure 3a). Five cases showed linear evolution from subclones (Figure 3b). Six cases demonstrated sweeping clonal evolution (Figure 3c). The evolution pattern of the remaining 24 patients either could not be defined by changes in mutations or VAF ( 4 cases), or harbored no candidate alterations before or after progression to AML.

6. Rivalry clonal evolution patterns were observed. Some original TRMs were replaced or outgrown by other mutations at sAML transformation, such as in patient 1 (UPN809); 6 (UPN3430), 10 (UPN4603), 18 (UPN2570), and 63 (UPN3567) (Table.1)

7. Although differences existed among TRMs, TET2/RUNX1/ASXL1/ DNMT3A/U2AF1/STAG2/ROB01/SF3B1 mutations were the most common initial/driver mutations for these AML-transformed patients (Table.2). However, ASXL1/BCORTET2/ mutations were most commonly accompanied by those transformation-related events when transformation occurred (Table.2).

\section{Whole exome sequencing}

Samples from three of the eight patients whose target sequencing showed no presumed TRMs but for whom sufficient DNA extract was still available were further subjected to whole exome sequencing (WES) (patients starred in Table 1). Figure 4 presents the results of WES from these cases. In addition to MDSrelated gene mutations, each gained at least one TRMs (involving transcription factor, or tumor suppressor genes) at the AML transformation point. Specifically, UPN1702 and UPN3831 acquired MLLT10 and NCOR2 mutations, respectively (both involved in transcription regulation), at the AML stage. MLLT10 is a histone lysine methyltransferase, which participates in AML pathogenesis via the formation of fusion gene MLLT10-MLL [14]. NCOR2 regulates gene transcription as a part of a histone deacetylases complex, and its dysfunction leads to functional abnormality in hematopoietic stem cells [15]. UPN3155 acquired a STK11 mutation (a tumor suppressor) after disease progression. It has been reported that STK11 regulates cell polarity and functions as a tumor suppressor [16].

\section{Single-cell RNA sequencing}

As mentioned above, abnormal cell signaling induced by gene mutation, such as RAS genes or PTPN11 may be critical for the transformation of MDS into AML. However, it is still unclear whether a RAS mutation is a requirement to activate RAS signaling pathways. To explore this question, we used single-cell RNA transcription sequencing to study the association between RAS mutation and RAS signaling in UPN4674 and UPN4763 (before and after disease progression). Two (NRAS and PTPN11 mutations) occurring during the AML stage are core genes in RAS signaling. As shown in Figure 5a and 5b, this patient presented several abnormal cell types (orange, UPN4674; blue, UPN4763 in Figure 5a). Gene classification analysis showed that aberrant granularmononuclear progenitors (GMP), common myeloid progenitor (CMP), megakaryocyte-erythroid progenitor (MEP) and monocytes (Mono) were present during the AML stage (Figure $5 b$ ). We focused on differences in the GMP population were RAS signaling. Integrated analysis based on single-cell sequencing indicated that several RAS signaling related-genes are expressed at high levels in GMP after disease progression (Figure 5c). These genes have been reported to participate in the activation of RAS signaling [17]. Similarly, RAS signaling related-genes such as FLT3, INSR, and CDC42 are also expressed at high levels in 
MEP, CMP, and Mono groups after disease progression (Figure 5d). These genes are closely associated with cell proliferation. Interestingly, apoptosis-related gene $B A D$ is down-regulated in GMP, MEP, CMP, and Mono groups after disease progression. These data suggest that transformation-related mutations gene mutations induce the redistribution of clonal cells, which leads to an increased number of morphological blasts and monocytes via the activation of cell signaling, and further leads to AML transformation.

\section{Discussion}

The pathogenesis of SAML differs from that of de novo AML in several respects including clinical progression and prognosis [18]. Despite poor response to contemporary therapies, including stem-cell transplantation, MDS-derived AML exhibits a relatively long pre-transformation duration, and occurs only in about one third of all MDS [7]. If this transformation requires triggering factors (defined as TRMs), and these factors are robust biomarkers of progression, it may be possible to develop target therapy.

In this study, we sequenced 72 paired bone marrow DNA samples, first at MDS diagnosis and then immediately after AML transformation. Sixty-four out of the 72 cases (88.9\%) were positive for TRMs, i.e., events involving active signaling, transcription factors, or tumor suppressors were checked out by target sequencing. Sequencing of 39 target genes could not detect any TRMs in eight out of the 72 cases. Of these, paired whole exome sequencing was performed for 3 cases for which sufficient DNA could be extracted from BM samples. Each of these patients carried at least one TRM (out of the 39 target genes), involving myeloid transcription, or tumor suppressor genes (Figure 4). No patient with MDS in this assay who developed sAML did so only by acquiring mutations involved in epigenetic modulation, RNA splicing, or cohesins. This finding suggests that the existence or emergence of certain last event-like mutations may be considered a prerequisite for MDS to transform into sAML, rather than just occasional or accompanying events [9, 10].

Among the 67 patients with detected TRMs, in 43 patients (64.2\%; including 40 by targeted sequencing and 3 by WES) the TRMs appeared to emerge at the point of AML transformation, and the remaining 24 patients harbored putative last mutations at the time of MDS diagnosis. Then it is necessary to tell which the original is? The existence/emerge of TRMs? Or the AML transformation itself. We considered that the pre-existence or emergence of TRMs should be the reason for AML transformation. First, for 24/67 (35.8\%) cases harbored TRMs at MDS diagnosis, the duration of transformation was moderately shorter in these cases than in those where the TRMs were only identified at the transformation point (mean 8 vs. 11 months). Second, due to the relatively longer corresponding intermittence between time points at diagnosis and AML transformation for paired sequencing, some important information may have been missed. When additional time points were added, supportive data were obtained from some patients (UPN1243, 3288, and 4390 in Figure 2). When TRMs emerged in these patients, their disease was still at the low-risk or pre-AML stage, but transformed to AML quickly. Finally, for one patient (UPN 4674), immediately after the occurrence of the AML phenotype, accompanied by NRAS and PTPN11 mutations, single-cell RNA sequencing showed both active RNA transcription and activation of the RAS/PTPN11 signaling pathway $[19,20]$. Given that the gene mutations occurred prior to RNA/protein transcription/translation, pathway activation, and ultimately, phenotype alteration. These results support our findings that TRMs and activated RAS signaling can precede phenotypic transformation to AML.

Most of the TRMs clones showed linear evolution from the founding clones (29 cases, compared to 5 cases with linear evolution in a pre-existing subclone; and 6 cases by clone sweeping) (Figure 3). This is somewhat different from published data [21, 22], may because of that a part of patients did not presented clonal progressing process, such as those 24 cases whose TRMs pre-existed at MDS diagnosis. In addition, we detected common pre-existing mutations involving TET2, RUNX1, ASXL1, DNMT3A, U2AF1, STAG2, ROBO1 and SF3B1 (Table.2), indicating that these mutations represent precursor mutations for these sAML cases. We ever reported poor prognosis for those MDS with ROBO mutations [23]. In view of the non-signaling/transcriptor/tumor suppressor function of ROBO, which may assist TRMs to finish AML transformation. As for ASXL1/BCOR/TET2 mutations, they emerged as common partners of TRMs (Table 2), possibly playing some roles in the transformation process. More research seemed necessary to explore the relationship between BCOR mutations (usually occur in patients with normal chromosomes) and sAML [24], especially for those sAML with RAS mutations as TRMs (Table.2). Some mutated genes such as RUNX1 (highly prevalent among patients with chromosome 7 abnormalities) [25] could be responsible either for the MDS phenotype or driving AML progression. These dynamic genetic features contribute to the complexity and heterogeneity of this disease and may become a key to deciphering the rule for sAML occurrence.

Together with TP53, mutations in NRAS/ KRAS, CEBPA, FLT3, CBL, PTPN11 and RUNX1 (total 7 genes) accounted for almost $90 \%$ patients for whom TRMs were defined by targeted sequencing. KIT mutation was never detected as only TRM for one patient in this group of cases, and NPM1 was only detected in one case. The latter could be attributed to the favorable responses of the MDS patients who harbor NPM1 mutations towards HMAs (decitabine), thus blocking sAML transformation [26]. Of note, we firstly reported CEBPA as one of the most common TRMs (accounting for nearly one-sixth of all TRMs), and expand the candidate genes for possible targeted treatment. In addition, the mutation pattern of FLT3 in this assay less involved FLT3-ITD (only in one of the 8 cases) (often observed in de novo AML), proposing the necessity to design point-mutations-aimed target therapy for patients with this kind of TRMs. In view of the rapid development of mutation-specific targeted therapy in recent years, we can hope to pharmacological block AML transformation in MDS patients at high transformation risk with regular monitoring for TRMs and administering effective corresponding targeted therapy.

In summary, somatic mutations in activated signaling, transcription factors, or tumor suppressor genes appear to be a precondition for AML transformation in myelodysplastic syndromes. The high propensity to acquire transformation-related gene mutations is worthy of further research as targets to be exploited with novel therapies.

\section{Conclusion}

We reported a large cohort of 72 cases of paired self-controlled data to identify the AML transformation- related mutations (TRMs). $88.9 \%$ of the 72 patients presented TRMs, which were mainly identified in a small set of genes including RAS, CEBPA, TP53, FLT3, etc. TRMs involving in signaling, transcription 
factors, or tumor suppressors appeared to be a precondition for AML transformation from MDS. The TRMs may be considered as new therapy targets.

\section{Declarations}

\section{Ethics approval and consent to participate}

All procedures in the current study involving human samples were performed in accordance with the Helsinki declaration and approved by hospital review boards of the Shanghai Jiao Tong University Affiliated Sixth People's Hospital. The informed consent has been obtained from each participant

\section{Consent for publication}

All authors have seen the manuscript and consented to publication.

\section{Availability of data and materials}

The data can be available by contacting the corresponding author.

\section{Competing interests}

There are no conflicts of interest to declare.

\section{Funding}

This work was supported by the National Natural Science Foundation of China (81770120 and 81770122 ).

\section{Authors' contributions}

X.L. was the principal investigators who conceived the study. F.X. and L.-Y.W. carried out most of the experiments. Q.H. was responsible for bioinformatics investigation. J.G. participated in the preparation of biological samples. Z.Z. helped gather detailed clinical information for the study and helped to carry out clinical analysis. X.L. and F.X. wrote the manuscript.

\section{Acknowledgments}

We thank Shanghai Tianhao Inc. for providing assistance in exome sequencing and data analysis. All authors are not employed by Shanghai Tianhao Inc., and declare that the research was conducted in the absence of any commercial or financial relationships that could be construed as a potential conflict of interest. We sincerely thank Dr. Rafael Bejar for providing constructive suggestion and revision.

\section{References}

1. Vetrie D, Helgason GV, Copland M. The leukaemia stem cell: similarities, differences and clinical prospects in CML and AML. Nat Rev Cancer. 2020 Mar; 20(3):158-173.

2. de Thé H, Chen Z. Acute promyelocytic leukaemia: novel insights into the mechanisms of cure. Nat Rev Cancer. 2010 Nov; 10(11):775-83.

3. Lin S, Mulloy JC, Goyama S. RUNX1-ETO Leukemia. Adv Exp Med Biol. 2017; 962:151-173.

4. Douet-Guilbert N, Chauveau A, Gueganic N et al. Acute myeloid leukaemia (FAB AML-M4Eo) with cryptic insertion of cbfb resulting in cbfb-Myh11 fusion. Hematol Oncol. 2017 Sep; 35(3):385-389.

5. Döhner H, Estey E, Grimwade D et al. Diagnosis and management of AML in adults: 2017 ELN recommendations from an international expert panel. Blood. 2017 Jan 26; 129(4):424-447.

6. Lindsley RC, Mar BG, Mazzola E et al. Acute myeloid leukemia ontogeny is defined by distinct somatic mutations. Blood. 2015 Feb 26; $125(9): 1367-76$.

7. Sperling AS, Gibson CJ, Ebert BL. The genetics of myelodysplastic syndrome: from clonal haematopoiesis to secondary leukaemia. Nat Rev Cancer. 2017 Jan; 17(1):5-19.

8. Raza A, Galili N. The genetic basis of phenotypic heterogeneity in myelodysplastic syndromes. Nat Rev Cancer. 2012 Dec; 12(12):849-59.

9. Walter MJ, Shen D, Ding L et al. Clonal architecture of secondary acute myeloid leukemia. N Engl J Med. 2012 Mar 22; 366(12):1090-8.

10. Kim T, Tyndel MS, Kim HJ et al. The clonal origins of leukemic progression of myelodysplasia. Leukemia. 2017 Sep; 31(9):1928-1935.

11. Li X, Xu F, Wu LY, et al. A genetic development route analysis on MDS subset carrying initial epigenetic gene mutations. Sci Rep. 2020 Jan $21 ; 10(1): 826$.

12. Vardiman JW, Thiele J, Arber DA et al. The 2008 revision of the World Health Organization (WHO) classification of myeloid neoplasms and acute leukemia: rationale and important changes. Blood. 2009; 114(5): 937-951.

13. Bennett JM, Catovsky D, Daniel MT et al. Proposals for the classification of the myelodysplastic syndromes. Br J Haematol. 1982; $51: 189-99$.

14. Van Limbergen H, Poppe B, Janssens A et al. Molecular cytogenetic analysis of 10;11 rearrangements in acute myeloid leukemia. Leukemia. 2002 Mar; 16(3):344-51.

15. Yan M, Burel SA, Peterson LF, Kanbe E et al. Deletion of an AML1-ETO C-terminal NcoR/SMRT-interacting region strongly induces leukemia development. Proc Natl Acad Sci U S A. 2004 Dec 7; 101(49):17186-91. 
16. Tarumoto Y, Lu B, Somerville TDD et al. LKB1, Salt-Inducible Kinases, and MEF2C Are Linked Dependencies in Acute Myeloid Leukemia. Mol Cell. 2018 Mar 15;69(6):1017-1027.e6.

17. Li S, Balmain A, Counter CM. A model for RAS mutation patterns in cancers: finding the sweet spot. Nat Rev Cancer. 2018 Dec;18(12):767-777.

18. Winer ES. Secondary Acute Myeloid Leukemia: A Primary Challenge of Diagnosis and Treatment. Hematol Oncol Clin North Am. 2020 Apr;34(2):449-463.

19. Quan X, Deng J. Core binding factor acute myeloid leukemia: Advances in the heterogeneity of KIT, FLT3, and RAS mutations (Review). Mol Clin Oncol. 2020 Aug;13(2):95-100.

20. Liu X, Ye Q, Zhao XP et al. RAS mutations in acute myeloid leukaemia patients: A review and meta-analysis. Clin Chim Acta. 2019 Feb; $489: 254-260$.

21. da Silva-Coelho P, Kroeze LI, Yoshida K et al. Clonal evolution in myelodysplastic syndromes. Nat Commun. 2017 Apr 21; 8:15099.

22. Makishima H, Yoshizato T, Yoshida K et al. Dynamics of clonal evolution in myelodysplastic syndromes. Nat Genet. 2017 Feb; $49(2): 204-212$.

23. Xu F, Wu LY, Chang CK et al. Whole-exome and targeted sequencing identify ROBO1 and ROBO2 mutations as progression-related drivers in myelodysplastic syndromes. Nat Commun. 2015 Nov 26; 6:8806.

24. Damm F, Chesnais V, Nagata Y et al. BCOR and BCORL1 mutations in myelodysplastic syndromes and related disorders. Blood. 2013 Oct 31 ; 122(18):3169-77.

25. Xu F, Wu LY, He Q, et al. Exploration of the role of gene mutations in myelodysplastic syndromes through a sequencing design involving a small number of target genes. Sci Rep. 2017 Feb 21; 7:43113.

26. Wu L, Li X, Xu F, et al. NPM1 mutation with DNMT3A wild type defines a subgroup of MDS with particularly favourable outcomes after decitabine therapy. Br J Haematol. 2020; 189(5):982-984.

\section{Tables}

Table.1 The results of target sequencing from paired samples of 72 patients 


\begin{tabular}{|c|c|c|c|c|c|}
\hline NoIUPN口 & Diagnosis & chromo & $\begin{array}{l}\text { MDS } \\
\text { to } \\
\text { AML) } \\
(\mathrm{m})\end{array}$ & Presumed last event & All gene mutations \\
\hline \multicolumn{6}{|c|}{$\begin{array}{l}\text { The patients in whom AML transformation-related mutations could be presumed by paired sequencing analysis } \\
\text { (listed as activated signaling, transcription factors and tumor suppressor } \\
\text { Activated signaling }\end{array}$} \\
\hline \multirow[t]{6}{*}{ 1.ه809ه } & RCMD & normal & & CEBPA/1/K352fs $\triangle 15 \square$ & ASXL1;CEBPA;EZH2; \\
\hline & & & & FLT3/9/D358V(48) & \\
\hline & & & & RUNX1/6/508+1G>C(24)/5/G170R(23) & \\
\hline & AML & normal & 17 & FLT3/9/D358V(48) & ASXL1; EZH2;FLT3;R \\
\hline & & & & RUNX1/6/508+1G>C(45)/5/G170R(42) & \\
\hline & & & & $N R A S / 2 G 13 R(48)$ & \\
\hline \multirow[t]{5}{*}{ 2.81057囚 } & RAEB1 & normal & & PTPRD/10/A539V\51囚 & DNMT3A; PTPRD;ST \\
\hline & AML & normal & 48 & PTPRD/10/A539V『46】 & DNMT3A; PTPRD;ST \\
\hline & & & & RUNX1/4/L98fs(28) & \\
\hline & & & & NRAS/2/3/G12V/Q61L(15)(15) & \\
\hline & & & & $C B L / 8 / C 384 R(12)$ & \\
\hline \multirow[t]{7}{*}{ 3. $82356 \rrbracket$} & RAEB1 & normal & & PHF6/4/C107fs(16) & BCOR;ITIH3;PHF6;RU \\
\hline & & & & RUNX1/5/A149_A150delinsGX囚16》 & \\
\hline & & & & $\mathrm{TP} 53 / 3 / \mathrm{c} .74+14 \mathrm{~T}>\mathrm{C}(51)$ & \\
\hline & AML & normal & 6 & PHF6/4/C107fs(23) & BCOR;ITIH3;PHF6;RU \\
\hline & & & & RUNX1/5/A149_A150delinsGX『21区 & \\
\hline & & & & $\mathrm{TP} 53 / 3 / \mathrm{c} .74+14 \mathrm{~T}>\mathrm{C}(55)$ & \\
\hline & & & & $K R A S / 2 / G 13 D(25)$ & \\
\hline \multirow[t]{4}{*}{ 4.(2998) } & RAEB2 & normal & & RUNX1/9/967+1>A6/886+1>A(31) & ASXL1;KRAS;RUNX1; \\
\hline & & & & KRAS/2/G12S(30) & \\
\hline & AML & normal & 2 & RUNX1/9/967+1>A6/886+1>A(28) & ASXL1;KRAS;RUNX1; \\
\hline & & & & $K R A S / 2 / G 12 S(27)$ & \\
\hline \multirow{2}{*}{$\begin{array}{l}5 . . \\
(2747)\end{array}$} & RAEB2 & normal & & & BCOR; ITIH3 \\
\hline & AML & normal & 10 & NRAS/2/G12D(57) & BCOR; ITIH3;KIF20B;I \\
\hline \multirow[t]{7}{*}{ 6.(3430) } & CMML1 & normal & & GATA2/4/G327E(29)/4/323_323del(13) & GATA2*2; KRAS;NRA \\
\hline & & & & RUNX1/6/D198Y(41) & \\
\hline & & & & KRAS/2/G12C(11) & \\
\hline & & & & NRAS/2/G12D(29) & \\
\hline & AML & normal & 7 & GATA2/4/G327E(29)/4/323_323del(38) & GATA2*2; KRAS; RUN \\
\hline & & & & RUNX1/6/D198Y(43) & \\
\hline & & & & $K R A S / 2 / G 12 C(45)$ & \\
\hline \multirow[t]{4}{*}{ 7.83435区 } & RCMD & normal & & RUNX1/1/P86S(46) & ASXL1;EZH2;RUNX1; \\
\hline & AML & normal & 33 & RUNX1/1/P86S(43) & ASXL1;EZH2;RUNX1; \\
\hline & & & & PTPN11/3/F71L(34) & \\
\hline & & & & NRAS/2/G12D(9.7) & \\
\hline \multirow[t]{3}{*}{ 8.83541区 } & RAEB2 & del20q- & & GATA2/5/L345W(43) & DNMT3A;GATA2;TET \\
\hline & AML & del20q- & 14 & GATA2/5/L345W(30) & DNMT3A;GATA2;TET \\
\hline & & & & KRAS/2/G12RD30I & \\
\hline
\end{tabular}




\begin{tabular}{|c|c|c|c|c|c|}
\hline \multirow[t]{3}{*}{ 9.『3626ه } & \multirow{3}{*}{$\begin{array}{l}\text { RCMD } \\
\text { AML }\end{array}$} & \multicolumn{3}{|l|}{ normal } & \multirow{3}{*}{$\begin{array}{l}\text { ASXL1;DHX9;IDH1;RI } \\
\text { ASXL1;DHX9;IDH1;RI }\end{array}$} \\
\hline & & NA & 29 & ETV6/6/G381fs(48) & \\
\hline & & & & $N R A S / 2 / G 12 A(44)$ & \\
\hline \multirow{5}{*}{$\begin{array}{l}10 \\
\varangle 4674 \rrbracket\end{array}$} & RCMD & Complex & & RUNX1/1/H78Y(53) & DNMT3A;ETV6;EZH2 \\
\hline & & & & ETV6/6/R339I(15) & \\
\hline & AML & Complex & 5 & RUNX1/1/H78Y(43】 & DNMT3A; KIF20B;RU \\
\hline & & & & NRAS/2/G12A(23) & \\
\hline & & & & PTPN11/3/A72V(18) & \\
\hline \multirow{5}{*}{$\begin{array}{l}11 . \\
\varangle 4687 \rrbracket\end{array}$} & RCMD & normal & & GATA2/3/M223I(51) & ASXL1;EZH2;GATA2; \\
\hline & & & & RUNX1/2/G138fs(42) & \\
\hline & AML & NA & 6 & GATA2/3/M223I(55) & ASXL1;EZH2;GATA2; \\
\hline & & & & RUNX1/2/G138fs(50) & \\
\hline & & & & NRAS/2/G12D(52) & \\
\hline \multirow{5}{*}{$\begin{array}{l}12 . \\
(4877)\end{array}$} & RCMD & normal & & MPL/2/A58V(47) & ASXL1;KRAS;MPL;S1 \\
\hline & & & & KRAS/2/G12S(41) & \\
\hline & AML & normal & 17 & MPL/2/A58V(46) & ASXL1;KRAS;MPL;S1 \\
\hline & & & & $K R A S / 2 / G 12 S(55)$ & \\
\hline & & & & PTPRD/23/R588C(52) & \\
\hline \multirow{3}{*}{$\begin{array}{l}13 . \\
(5305)\end{array}$} & RCMD & normal & & NRAS/2/G13D(4.1)/2/G12D(3.4) & IDH1;KMT2D;NRAS; \\
\hline & AML & normal & 9 & $N R A S / 2 / G 13 D(38)$ & IDH2;KMT2D;NRAS;E \\
\hline & & & & ETV6/4/H141Tfs313(16) & \\
\hline \multirow{4}{*}{ 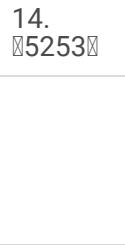 } & RCMD & normal & & None & None \\
\hline & AML & $t(3 ; 6),-7$ & 40 & RUNX1/6/R201Q(48) & ANKRD11;NRAS;PTP \\
\hline & & & & NRAS/3/Q61H(25) & \\
\hline & & & & PTPN11/3/E69K(23.4) & \\
\hline \multirow{4}{*}{$\begin{array}{l}15 . \\
(1587)\end{array}$} & RAEB1 & der2 & & JAK2/14/V617F(46) & IDH2;FLT3;JAK2;U2A \\
\hline & & & & FLT3/19/c.2208-14A.G(52) & \\
\hline & AML & der2 & 25 & JAK2/14/V617F(43) & IDH2;FLT3;JAK2;U2A \\
\hline & & & & FLT3/19/c.2208-14A.G(49) & \\
\hline \multirow{3}{*}{$\begin{array}{l}16 . \\
(1792)\end{array}$} & RAEB1 & der4 & & RUNX1/9/Q397R』47凶 & RUNX1;U2AF1 \\
\hline & AML & der4 & 8 & RUNX1/9/Q397R囚30》 & RUNX1;U2AF1; FLT3 \\
\hline & & & & FLT3/11/E444V(37)11/E444X)(37) & \\
\hline \multirow{3}{*}{$\begin{array}{l}17 . \\
(2117)\end{array}$} & RCMD & complex & & TP53/5/8/Y126C(16)/R273S(15) & SRSF2;TP53*2 \\
\hline & AML & complex & 8 & TP53/5/8/Y126C(14)/R273S(14) & TP53*2;FLT3 \\
\hline & & & & FLT3/9/D358V(55) & \\
\hline \multirow{2}{*}{$\begin{array}{l}18 . \\
(2570)\end{array}$} & RAEB1 & normal & & RUNX2/6/D198N(35) & IDH2;RUNX1;SRSF2 \\
\hline & AML & +8 & 5 & FLT3/11/A445V(49)/11/A445S(49) & FLT3*2; \\
\hline \multirow{5}{*}{$\begin{array}{l}19 . \\
(2577)\end{array}$} & CMML1 & normal & & CEBPA/1/p69-70del(17) & CEBPA;DNMT3A;NPN \\
\hline & & & & NPM1/10/L258fs(33) & \\
\hline & AML & normal & 11 & CEBPA/1/p69-70del(21) & CEBPA;DNMT3A;NPN \\
\hline & & & & NPM1/10/L258fs(25) & \\
\hline & & & & FLT3/14/K602delinsREYEYDLK (14) & \\
\hline
\end{tabular}




\begin{tabular}{|c|c|c|c|c|c|}
\hline \multirow{4}{*}{$\begin{array}{l}20 . \\
(3183)\end{array}$} & RCMD & $\operatorname{add}(3)+8$, & & & BCOR;DHX9;EZH2*2; \\
\hline & \multirow[t]{3}{*}{ AML } & \multirow[t]{3}{*}{$\operatorname{add}(3)+8}$, & \multirow[t]{3}{*}{6} & \multirow{3}{*}{$\begin{array}{l}F L T 3 / 9 / D 358 V(52) \\
C B L / 11 / C 1564-13 C>T(40)\end{array}$} & BCOR; DHX9; EZH2*。 \\
\hline & & & & & \\
\hline & & & & & TET2 \\
\hline \multirow{5}{*}{$\begin{array}{l}21 . \\
(4383)\end{array}$} & RCMD & normal & & & DNMT3A;SF3B1 \\
\hline & AML & $+1, \operatorname{der}(1 ; 16)$ & 18 & PHF6/2/S22ð(5.6) & DNMT3A;SF3B1;CBL \\
\hline & & & & FLT3/9/T382M(45) & \\
\hline & & & & $C B L / 15 / E 765 G(49)$ & \\
\hline & & & & $K I T / 14 / S 713 F(49)$ & \\
\hline \multirow{2}{*}{$\begin{array}{l}22 . \\
(5135)\end{array}$} & RAEB2 & Add7,del20q & & & ASXL1;ITIH3;TET2;U: \\
\hline & AML & Add7,del20q & 5 & FLT3-ITDDhigh"1.597 & ASXL1;ITIH3;TET2;U: \\
\hline \multirow[t]{7}{*}{ 23.(692) } & CMML2 & normal & & GATA2/3/S186T(47) & GATA2;ITIH3;PHF6;R \\
\hline & & & & PHF6/9/:c.968+1G>A囚25囚 & \\
\hline & & & & RUNX1/9/F416fs(27) & \\
\hline & AML & normal & 10 & GATA2/3/S186T(50) & GATA2;ITIH3;PHF6;R \\
\hline & & & & PHF6/9/:c.968+1G>A囚26ه & \\
\hline & & & & RUNX1/9/F416fs(24) & \\
\hline & & & & $C B L / 9 / R 420 Q(34)$ & \\
\hline \multirow{3}{*}{$\begin{array}{l}24 . \\
(1891)\end{array}$} & CMML1 & der22 & & & DNMT3A;ROBO2;TEר \\
\hline & AML & der22 & 14 & $C B L / 11 / c .1564-13 C>T(56)$ & DNMT3A;ROBO2;TEך \\
\hline & & & & CEBPA $1 / A 265 f s(37) K 90 f s(52)$ & \\
\hline \multirow{6}{*}{$\begin{array}{l}25 . \\
(2206)\end{array}$} & RCMD & +8 & & NF1/17/M645V(49) & ANKRD11;ASXL1;CB \\
\hline & & & & PTPRD/29A1047S(44) & \\
\hline & & & & $\mathrm{CBL} / 8 / \mathrm{C} 396 \mathrm{R}(27 \mathrm{D}$ & \\
\hline & AML & +8 & 3 & NF1/17/M645V(51) & ANKRD11;ASXL1;CB \\
\hline & & & & PTPRD/29A1047S(40) & \\
\hline & & & & $C B L / 8 / C 396 R(21)$ & \\
\hline \multirow{2}{*}{$\begin{array}{l}26 . \\
(4559)\end{array}$} & RAEB2 & complex & & CBL/15/S791fs(9.6)/15/L790fs(9.7) & ANKRD11;ASXL1;CB \\
\hline & AML & complex & 11 & CBL/15/S791fs(8.7)/15/L790fs(8.8) & ANKRD11;ASXL1;CB \\
\hline \multirow{2}{*}{$\begin{array}{l}27 . \\
(2638)\end{array}$} & CMML1 & normal & & PTPN11/14/P563R(43) & PTPN11; ROB01; \\
\hline & AML & normal & 16 & PTPN11/14//P563R(45) /3/D61V(37) & PTPN11×2; ROBO1; \\
\hline \multirow{5}{*}{$\begin{array}{l}28 . \\
(4133)\end{array}$} & RAEB1 & complex & & CBL/9/R420Q(71) & ASXL1;CBL;ROBO2;T \\
\hline & & & & TP53/4/c.306-2A>G(96) & \\
\hline & AML & complex & 3 & CBL/9/R420Q(39) & ASXL1;CBL;ROBO2;T \\
\hline & & & & TP53/4/c.306-2A>G(97) & \\
\hline & & & & PTPN11/13/G507V(28) & \\
\hline \multirow{2}{*}{$\begin{array}{l}29 . \\
(4410)\end{array}$} & RAEB1 & del20q & & PTPN11/3/D61H(33) & EZH2; PTPN11;SETB \\
\hline & AML & del20q & 12 & PTPN11/3/D61H(48) & EZH2; PTPN11;SETB \\
\hline \multirow{2}{*}{$\begin{array}{l}30 \\
\varangle 4820 \rrbracket\end{array}$} & RCMD & Add3,add18 & & & $\mathrm{EZH2;KMT2D;U2AF1}$ \\
\hline & AML & Add3 & 15 & PTPN11/3/E76A(8.0) & $\mathrm{EZH} 2 ; \mathrm{KMT} 2 \mathrm{D} ; \mathrm{U} 2 \mathrm{AF} 1$ \\
\hline $\begin{array}{l}31 . \\
(3891)\end{array}$ & RCMD & normal & & PTPRD/18/I930V『51ه & ASXL1;PTPRD \\
\hline
\end{tabular}




\begin{tabular}{|c|c|c|c|c|c|}
\hline & AML & normal & 8 & PTPRD/18/I930V(46) & ASXL1;PTPRD \\
\hline \multirow[t]{3}{*}{ 32.(702) } & RCMD & der16,(1;16)del20q & & & ANKRD11;SRSF2 \\
\hline & AML & $\operatorname{der} 16 t(1 ; 16)$ del20q & 28 & MPL/10/W515L(17) & ANKRD11;SRSF2هGA \\
\hline & & & & GATA2/6/R396W(15) & \\
\hline \multicolumn{6}{|c|}{ Myeloid Transcriptors } \\
\hline \multirow{3}{*}{$\begin{array}{l}33 . \\
\text { (1018) }\end{array}$} & RAEB2 & derX,der14 & & & DNMT3A; EZH2; TET \\
\hline & AML & derX,der14 & 4 & FLT3:/9/D358V(53) & DNMT3A; EZH2; TET \\
\hline & & & & CEBPA1/A295V(39) & \\
\hline \multirow{3}{*}{$\begin{array}{l}34 . \\
(1090)\end{array}$} & RARS & del20q & & RUNX1/9/D344G(45囚 & DNMT3A囚DHX9هRUP \\
\hline & AML & del20q & 11 & RUNX1/9/D344G(48)/7/R232W(20) & DNMT3A囚 DHX9هRU \\
\hline & & & & CEBPA $1 / Y 285 f s(32)$ & SEIBPI \\
\hline \multirow{2}{*}{$\begin{array}{l}35 . \\
(1243)\end{array}$} & RCMD & normal & & & ASXL1; U2AF1 \\
\hline & AML & normal & 18 & CEBPAV1/7_15del[140! & ASXL1; U2AF1; CEBF \\
\hline \multirow{2}{*}{$\begin{array}{l}36 \\
(1931)\end{array}$} & RAEB1 & complex & & CEBPA $1 / S 9 F(67)$ & CEBPA;DNMT3A;TET \\
\hline & AML & complex & 9 & CEBPA $1 / S 9 F(46)$ & CEBPA;DNMT3A;TET \\
\hline \multirow{2}{*}{$\begin{array}{l}37 . \\
(3288)\end{array}$} & $\mathrm{RN}$ & normal & & & KIF20B囚STAG2囚TET: \\
\hline & AML & normal & 12 & CEBPA $1 / L 338 P(36)$ & KIF20B囚STAG2囚TET: \\
\hline \multirow{2}{*}{$\begin{array}{l}38 . \\
(3552)\end{array}$} & RARS & $-Y$ & & & SF3B1; TET2 \\
\hline & AML & NA & 28 & CEBPA1/c238delineCSG(18) & SF3B1; TET2; CEBPA \\
\hline \multirow{2}{*}{$\begin{array}{l}39 . \\
(3826)\end{array}$} & RCMD & del7 & & & ASXL1;EZH2 \\
\hline & AML & normal & 22 & CEBPA $1 / Y 166 X(48) P 58 f s(47)$ & ASXL1;EZH2;CEBPA* \\
\hline \multirow{6}{*}{$\begin{array}{l}40 . \\
(4196)\end{array}$} & RCMD & del20q,+21 & & PHF6/4/G93S(27) & PHF6; RUNX1;SRSF2 \\
\hline & & & & RUNX1/1/R80C(51) & \\
\hline & AML & del20q,+21 & 8 & PHF6/4/G93S(45) & PHF6; RUNX1;SRSF2 \\
\hline & & & & RUNX1/1/R80C(78) & \\
\hline & & & & NRAS/2/G12D『34囚 & \\
\hline & & & & CEBPA1/69_70del(11) & \\
\hline \multirow{5}{*}{$\begin{array}{l}41 . \\
(4634)\end{array}$} & RAEB2 & normal & & NPM1/7/L160fs(24) & NPM1 $₫ N R A S \llbracket S F 3 B 1$ \\
\hline & & & & NRAS/2/G13D(16) & \\
\hline & AML & normal & 2 & NPM1/7/L160fs(35) & NPM1 $₫ N R A S \llbracket S F 3 B 1$ \\
\hline & & & & NRAS/2/G13D(37) & \\
\hline & & & & CEBPAV1/69_70del(9.8) & \\
\hline \multirow{2}{*}{ 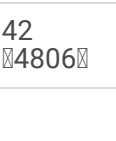 } & CMML1 & normal & & & ANKRD11;DHX9;TET \\
\hline & AML & +8 & 11 & CEBPA $1 / 1 / E 228 f s(4.8) / L 212 Q(28)$ & ANKRD11;DHX9;TET \\
\hline \multirow{4}{*}{$\begin{array}{l}43 . \\
(1350)\end{array}$} & RAEB1 & complex & & PHF6/9/Y303X(56) & EZH2;PHF6;RUNX1;T \\
\hline & & & & RUNX1/9/P333fs(29) & \\
\hline & AML & complex & 2 & PHF6/9/Y303X(46) & EZH2;PHF6;RUNX1;T \\
\hline & & & & RUNX1/9/P333fs(17) & \\
\hline \multirow{2}{*}{$\begin{array}{l}44 . \\
(1582)\end{array}$} & CMML1 & normal & & PTPRD/22/c.962-9C>G(47) & KIF20B;PTPRD;SF3B \\
\hline & AML & normal & 13 & PTPRD/22/c.962-9C>G(43) & KIF20B;PTPRD;SF3B \\
\hline
\end{tabular}




\begin{tabular}{|c|c|c|c|c|c|}
\hline 45. & RCMD & normal & & RUNX1/3/R174Q(28) & ASXL1;RUNX1;TET2; \\
\hline & AML & normal & 15 & RUNX1/3/R174Q(42) & ASXL1;RUNX1;TET2’' \\
\hline 46. & RCMD-RS & normal & & RUNX1/4/H105Y $₫ 40 \rrbracket$ & ASXL1;ROB01;RUNX \\
\hline & AML & del5q & 3 & 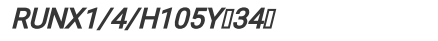 & ASXL1;ROB01;RUNX \\
\hline 47. & RAEB1 & normal & & RUNX1/4/S229fs『34凹 & BCOR;DNMT3A;IDH2 \\
\hline & AML & normal & 3 & RUNX1/4/S229fs】39] & BCOR;DNMT3A;IDH2 \\
\hline 48.(811) & RCMD & der6,t $(1 ; 6)$ & & & None \\
\hline & AML & der6,t $(1 ; 6)$ & 26 & ETV6/6/V345LD52ם & ETV6;MPL \\
\hline & & & & $M P L / 10 / W 515 R(50)$ & \\
\hline 49. & $\mathrm{RCMD}$ & normal & & ETV6/7/M389R(33) & ASXL1;BCOR;ETV6;P \\
\hline & & & & PHF6/10/R342X(27) & \\
\hline & AML & inv3add3 & 15 & PHF6/10/R342X(45) & BCOR;ETV6;PHF6 \\
\hline & & & & ETV6/7/M389R(42) & \\
\hline 50. & RAEB2 & normal & & SETBP1/3/c487-3C>T(44) & SETBP1 \\
\hline & AML & normal & 8 & SETBP1/3/c487-3C>T(43) & SETBP1 \\
\hline 51. & RAEB2 & normal & & & STAG2;UPF3A \\
\hline & AML & normal & 40 & SETBP1/4/D868N(18) & SETBP1;SRSF2 \\
\hline & Tum & suppressor & & & \\
\hline 52.(843) & RCMD & der1del1 & & & SRSF2; TET2 \\
\hline & AML & der1 & 3 & TP53/5/C135X(18) & SRSF2;TET2;TP53 \\
\hline 53. & RAEB1 & normal & & & None \\
\hline & AML & complex & 7 & TP53/8/E287fs(40)/6/G199E(28) & ASXL1هTP53×2 \\
\hline 54. & RAEB2 & normal & & TP53/8/E287D(56) & ROB01;TP53;UPF3A \\
\hline & AML & del11q & 27 & TP53/8/E287D[48D & ROB01;TP53;UPF3A \\
\hline 55. & RAEB2 & complex & & TP53/7/M237I(74) & DNMT3A; TP53 \\
\hline & AML & complex & 3 & TP53/7/M237I(80) & DNMT3A; TP53 \\
\hline 56. & RAEB1 & complex & & TP53/4/W91X(30) & ANKRD11;DNMT3A; \\
\hline & AML & complex & 13 & TP53/4/W91X(30)/5/R158L(31) & ANKRD11;DNMT3A; \\
\hline 57. & RAEB2 & normal & & & DHX9; ROB01; \\
\hline & AML & normal & 4.5 & TP53/9/R303fs(85) & DHX9;ROB01; TP53 \\
\hline 58. & RAEB1 & normal & & PTPRD/27/G809V(56) & DNMT3A;IDH2;PTPR \\
\hline & AML & normal & 18 & PTPRD/27/G809V(51) & DNMT3A;IDH2;PTPR \\
\hline & & & & TP53/5/P151S(25) & \\
\hline 59. & RAEB1 & complex & & TP53/5/530-535del (34) & TP53 \\
\hline & AML & complex & 15 & TP53/5/530-535del053ם & TP53 \\
\hline 60. & CMML1 & complex & & TP53/2/S83G(84) & TP53 \\
\hline & AML & complex & 13 & TP53/2/S83G(60) & TP53 \\
\hline
\end{tabular}




\begin{tabular}{|c|c|c|c|c|c|}
\hline \multirow{4}{*}{$\begin{array}{l}61 . \\
(1670)\end{array}$} & \multirow[t]{4}{*}{ RCMD } & \multirow[t]{4}{*}{ del18q } & & GATA2/5/M388fs(41) & \multirow[t]{4}{*}{ BCOR;ETV6;GATA2;T } \\
\hline & & & & TP53/2/E11Q(51) & \\
\hline & & & & ETV6/5/V285M(49) & \\
\hline & & & & WT1/1/A115fs(12) & \\
\hline & \multirow[t]{4}{*}{ AML } & \multirow[t]{4}{*}{ del18q } & \multirow[t]{4}{*}{2} & GATA2/5/M388fs(40) & \multirow[t]{4}{*}{ BCOR;ETV6;GATA2;T } \\
\hline & & & & TP53/2/E11Q(50) & \\
\hline & & & & ETV6/5/V285M(53) & \\
\hline & & & & WT1/1/A115fs(25) & \\
\hline \multirow{2}{*}{$\begin{array}{l}62 . \\
(1849)\end{array}$} & RCMD & normal & & WT1/7/H428fs『31】 & DNMT3A;IDH1;TET2; \\
\hline & AML & normal & 8 & WT1/7/H428fs $\square 40 \square$ & DNMT3A;IDH1;TET2 \\
\hline \multirow{4}{*}{$\begin{array}{l}63 . \\
(3567)\end{array}$} & \multirow[t]{2}{*}{ RAEB2 } & \multirow[t]{2}{*}{ normal } & & FLT3/12/c.1419-3->T(15) & \multirow[t]{2}{*}{ DHX9;DNMT3A;ANKI } \\
\hline & & & & NPM1/10/R262fs(43)/10W259delinsWQ(44)/10/R262S(44)/7/W163L(42) & \\
\hline & \multirow[t]{2}{*}{ AML } & \multirow[t]{2}{*}{ normal } & \multirow[t]{2}{*}{4} & NPM1/10/R262fs(12)/10W259delinsWQ(25)/10/R262S(19)/7/W163L(20) & \multirow[t]{2}{*}{ DHX9;DNMT3A;ANKI } \\
\hline & & & & WT1/7/S169fs(32) & \\
\hline \multirow{2}{*}{$\begin{array}{l}64 . \\
(1892)\end{array}$} & RAEB2 & normal & & NPM1/11/L287f囚35区 & DNMT3A;IDH1;NPM¹ \\
\hline & AML & normal & 9 & NPM1/11/L287fロ31D & DNMT3A;IDH1;NPM1 \\
\hline \multicolumn{6}{|c|}{ The patients in whom last gene events could not be presumed by paired target sequencing analysis } \\
\hline \multirow{2}{*}{$\begin{array}{l}65 . \\
(1075)\end{array}$} & RA & normal & & & DHX9;IDH2;ROBO2; \\
\hline & AML & normal & 20 & & DHX9;IDH2;ROBO2; ؟ \\
\hline \multirow{2}{*}{$\begin{array}{l}66 . \\
(1619)\end{array}$} & RAEB2 & complex & & & DHX9;DNMT3A;ITIHE \\
\hline & AML & complex & 25 & & DHX9;DNMT3A;ITIHÉ \\
\hline \multirow{2}{*}{$\begin{array}{l}67 . \\
(1702) \rrbracket\end{array}$} & RAEB2 & normal & & & SRSF2 \\
\hline & AML & der12 & 19 & & SRSF2 \\
\hline \multirow{2}{*}{$\begin{array}{l}68 . \\
(3831) \rrbracket\end{array}$} & RAEB2 & normal & & & ANKRD11;IDH2 \\
\hline & AML & normal & 4 & & ANKRD11;IDH2 \\
\hline \multirow{2}{*}{$\begin{array}{l}69 . \\
(3155) \rrbracket\end{array}$} & RCMD & +14, del20q & & & ROB01 \\
\hline & AML & normal & 7.5 & & ROB01 \\
\hline \multirow{2}{*}{$\begin{array}{l}70 . \\
(3437)\end{array}$} & RA & Inv9 & & & DNMT3A;ROB01 \\
\hline & AML & Inv9 & 3 & & DNMT3A;ROB01 \\
\hline \multirow{2}{*}{$\begin{array}{l}71 . \\
(4145)\end{array}$} & RCMD & $+1, \operatorname{der}(1: 7)$ & & & EZH2;IDH1 \\
\hline & AML & NA & 14 & & ASXL1;TET2*2खZRSI \\
\hline \multirow{2}{*}{$\begin{array}{l}72 . \\
\square 4404 \rrbracket\end{array}$} & RAEB1 & complex & & & SRSF2;TET2 \\
\hline & AML & complex & 18 & & TET2 \\
\hline
\end{tabular}

Notes:1. The fifth column indicates the possible last event mutations. The mutations with italic and bold font were shown as the final presumed AML transformation-related mutations (TRMs) according to the definition in the METHOD part. 2. The paired target sequencing analysis from the last eight patients could not define the TRMs, so three (with star signals) of them accepted the whole exome sequencing to look for possible TRMs which were not included in 39 target genes. For explanation of mutation type, splicing site mutation was shown in nucleotide variation. 3. NA means no available data.

Table 2. Characteristics summary of the top seven TRMs by target sequencing 


\begin{tabular}{|c|c|c|c|c|}
\hline TRMs & cases & $\begin{array}{l}\text { Emerged } \\
\text { at } \\
\text { sAML(n) }\end{array}$ & $\begin{array}{l}\text { Primary } \\
\text { diagnosis } \geq \text { RAEB2/CMML2(n) }\end{array}$ & Most common earlier mutations at MDS diagnosis(top 2-3)(top5 in Total)(n) \\
\hline NRAS/KRAS & $14(5)$ & $10 / 14$ & $3 / 14$ & RUNX1(8)/ASXL1(6)TET2(6)STAG2(5) \\
\hline CEBPA & $11(1)$ & $10 / 11$ & $2 / 11$ & TET2(7)/DNMT3A(4)/ASXL1(2)EZH2(2邓SF3B1(2)RUNX1(2)SRSF2(2)U2AF1(2) \\
\hline TP53 & 9 & $5 / 9$ & $3 / 9$ & DNMT3A(3)/ROBO1(2) \\
\hline FLT3 & $8(2)$ & $7 / 8$ & $1 / 8$ & U2AF1(4)/IDH2(2)RUNX1(2)SRSF2(2)DNMT3A(2)ROBO1(2)TET2(2) \\
\hline$C B L$ & $7(3)$ & $5 / 7$ & $2 / 7$ & $\begin{array}{l}\text { DNMT3A(3)/U2AF1(3)/ANKRD11(2)ASXL } \\
\text { 1(2)STAG2(2)/PTPRD(2)/ROBO1(2)/SF3B1(2) }\end{array}$ \\
\hline PTPN11 & $6(2)$ & $4 / 6$ & $0 / 6$ & EZH2(3)SETBP1(2) \\
\hline RUNX1 & 5 & $1 / 5$ & $0 / 5$ & TET2(3)/ASXL1(2)SF3B1(2) \\
\hline Total & $60(13)$ & $42 / 60$ & $11 / 60$ & TET2(18)/RUNX1(12)/ASXL1(12)DNMT3A(12)/U2AF1(9)/STAG2(7)ROB01(6)SF3B \\
\hline
\end{tabular}

NOTE: 1.The TRMs ( $\leq 3$ cases in total) are not included in this Table. 2 . The number in the brackets of the second column represented the cases that had another presumed TRMs besides the mainly presumed TRMs. 3 . The mutations listed in the last column means the mutations which emerged together with the presumed TRMs, may playing roles during the MDS-sAML transformation.

\section{Figures}

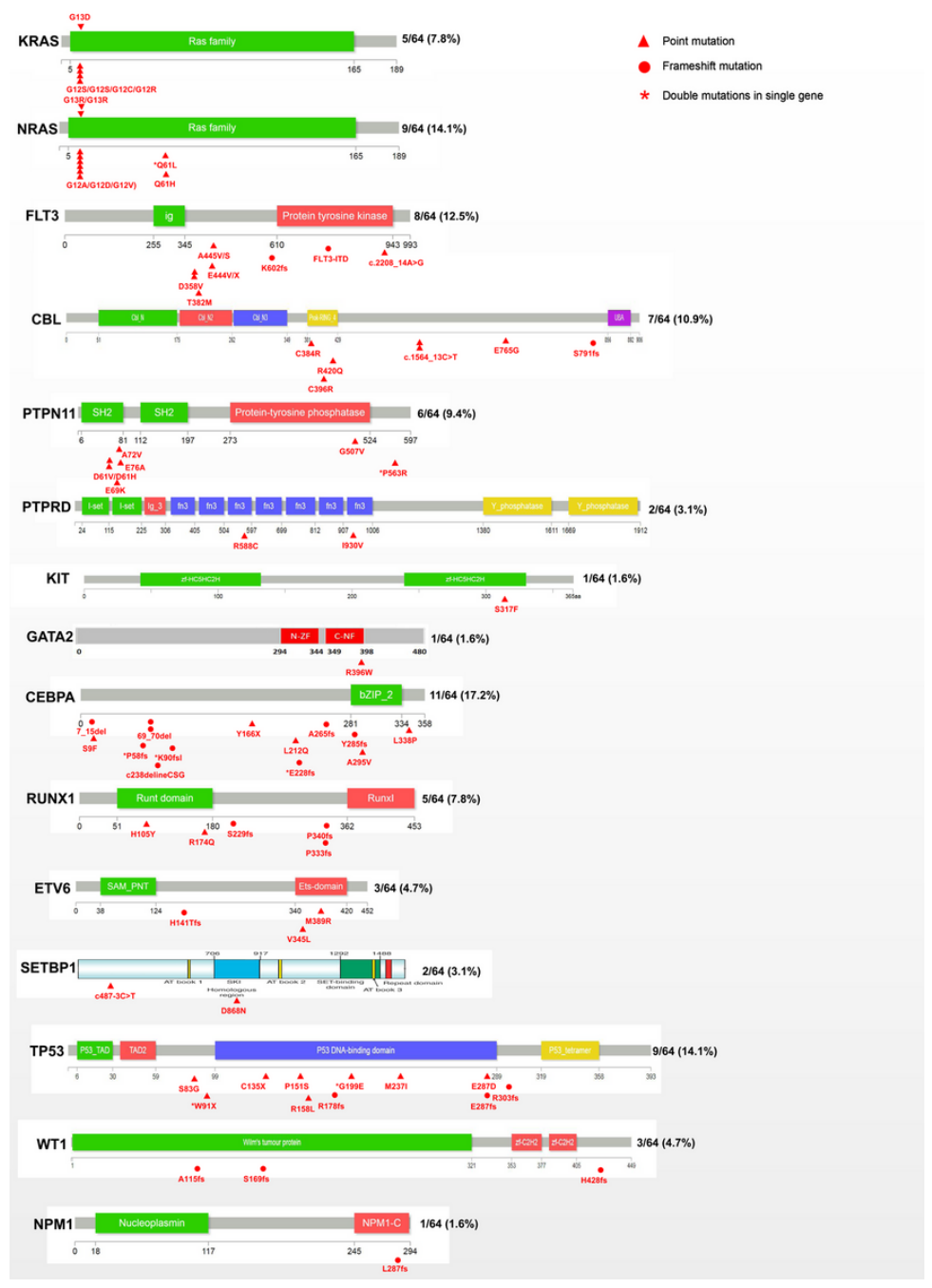

Figure 1

\section{Occurrence frequency and sites of last mutation events}

All mutations, including types and sites, are shown for $\mathbf{1 3} \mathrm{AML}$ transformation-related mutations, according to the definitions. Triangles and circles indicate point and frameshift mutations, respectively. Stars indicate double mutations occurring in a single gene. 

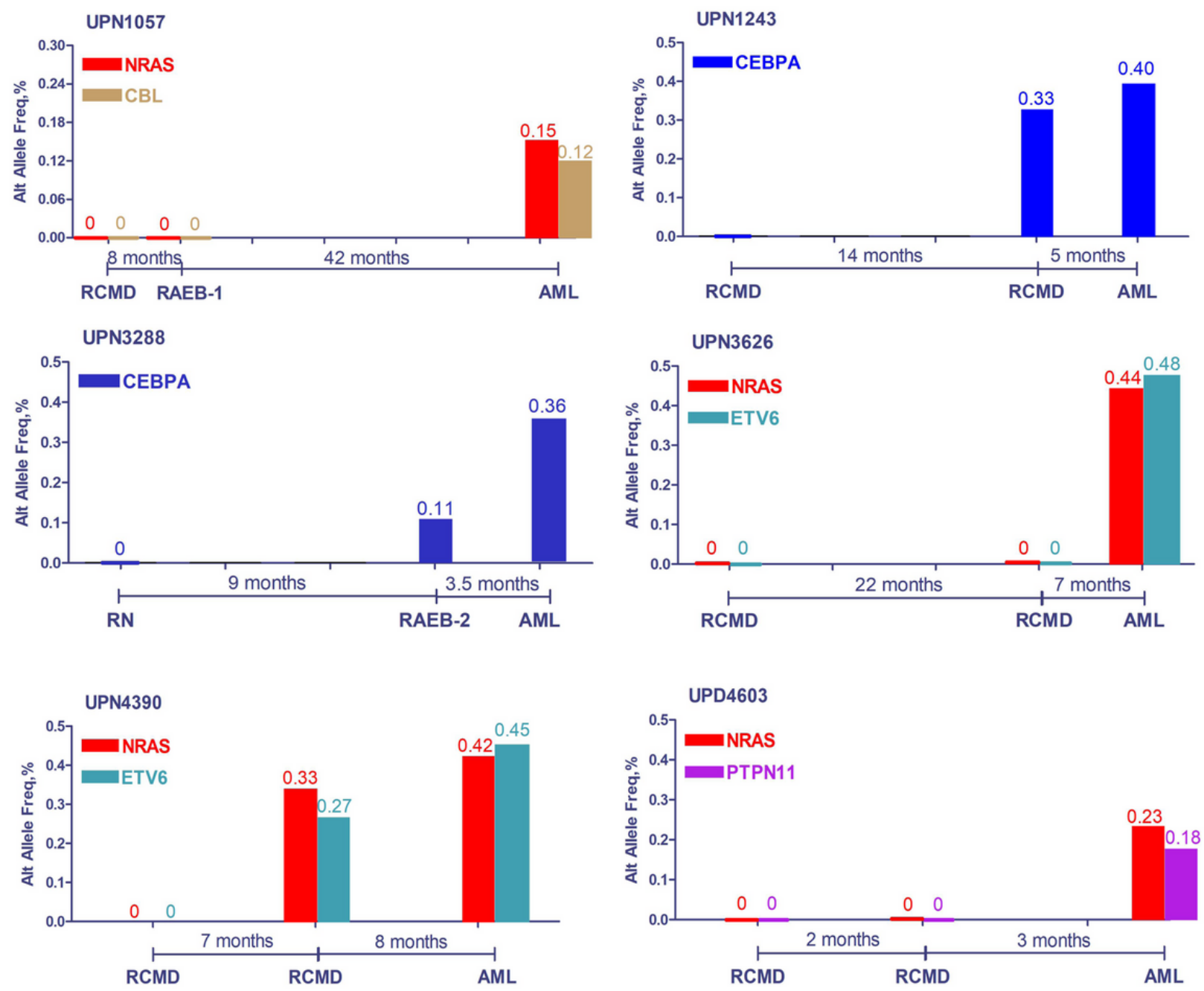

Figure 2

Evolution route for six cases that were analyzed by an additional sequencing assay between MDS diagnosis and AML transformation 


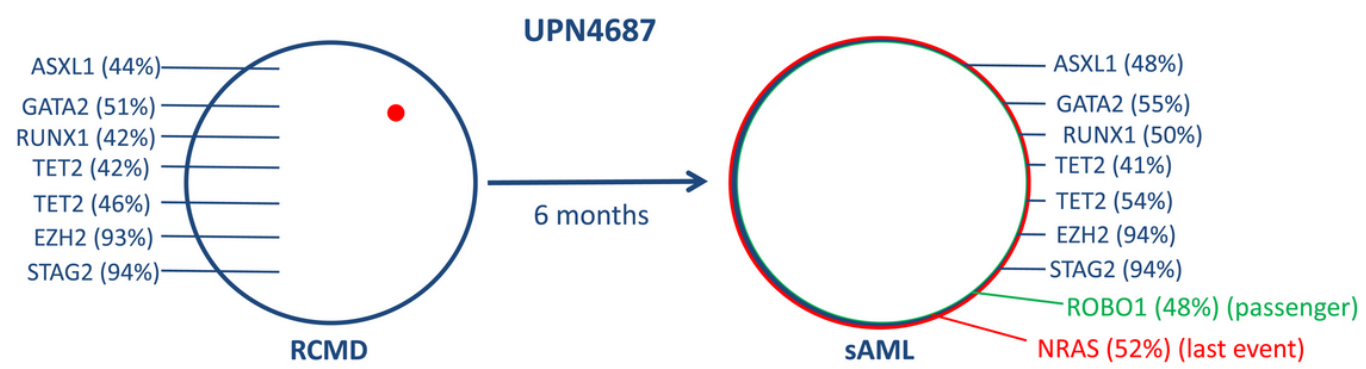

b

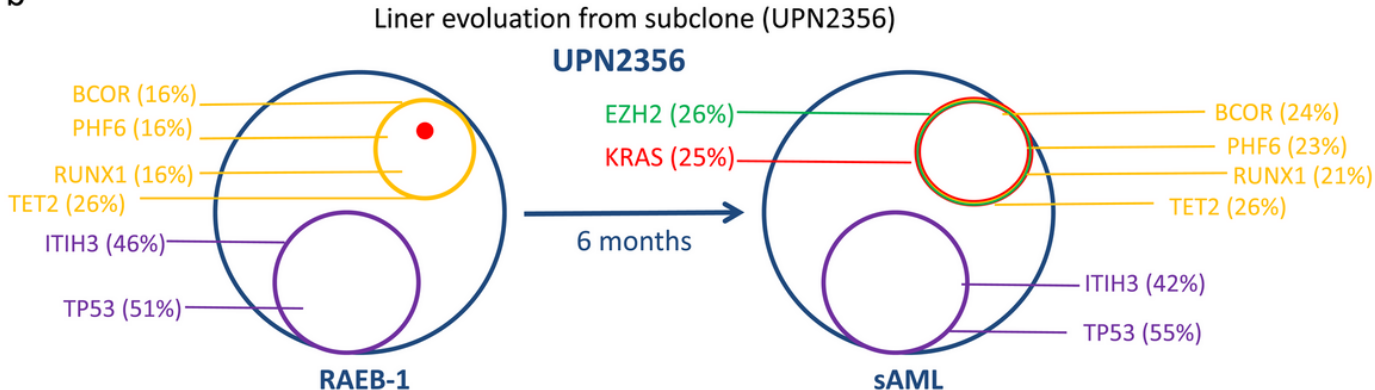

C

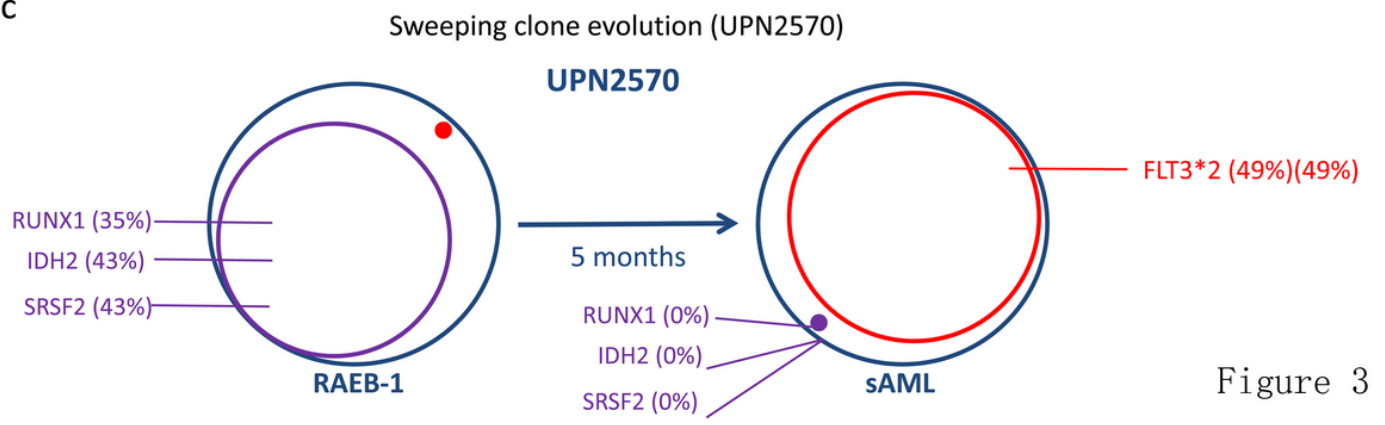

\section{Figure 3}

Three evolution patterns for AML transformation

a. Linear evolution, based on the founding clone, is shown for UPN4687. Similar cases include UPN809, 1057, 3435, 3626, 1792, 3183, 1891, 1243, 3288, 1090, $1018,3552,3826,4196,4634,1582,1831,2296,843,702,3430,3541,2638,4133,4820,4877,5135$, and 5305 . b. Linear evolution from a subclone is shown for UPN2356. Similar cases include UPN4603, 2577, 692, and 2666. Liner evoluation includes two models (from founding clone and subclone). Red dots mean the origin of red cycle (right graph). c. Sweeping clone evolution is shown for UPN2570. Similar cases include UPN811, 1354, 3053, 3567 and 4383. [Number] indicates VAF; $[\mathrm{H}]$ indicates homozygous mutations; red indicates presumed last mutation; green indicates newly emerged partner mutations, which could not be presumed to be AML transformation-related mutations. 

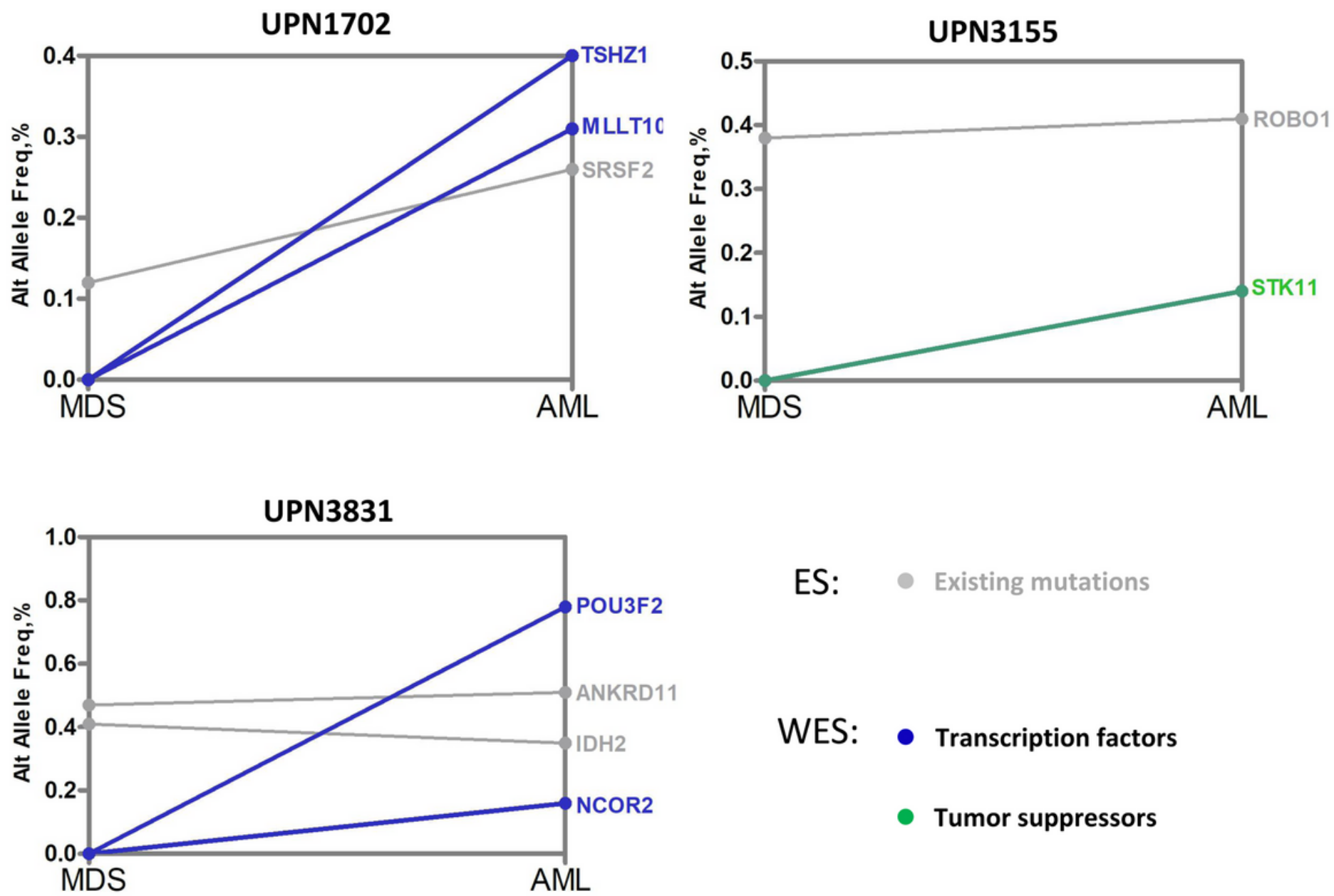

Figure 4

Whole exome sequencing revealed other atypical last mutation events

Three patients showed negative results for typical AML transformation-related mutations. However, whole exome sequencing revealed some other atypical AML transformation-related mutations. Gray indicates existing mutations; blue indicates transcription factors; green indicates tumor suppressors. 

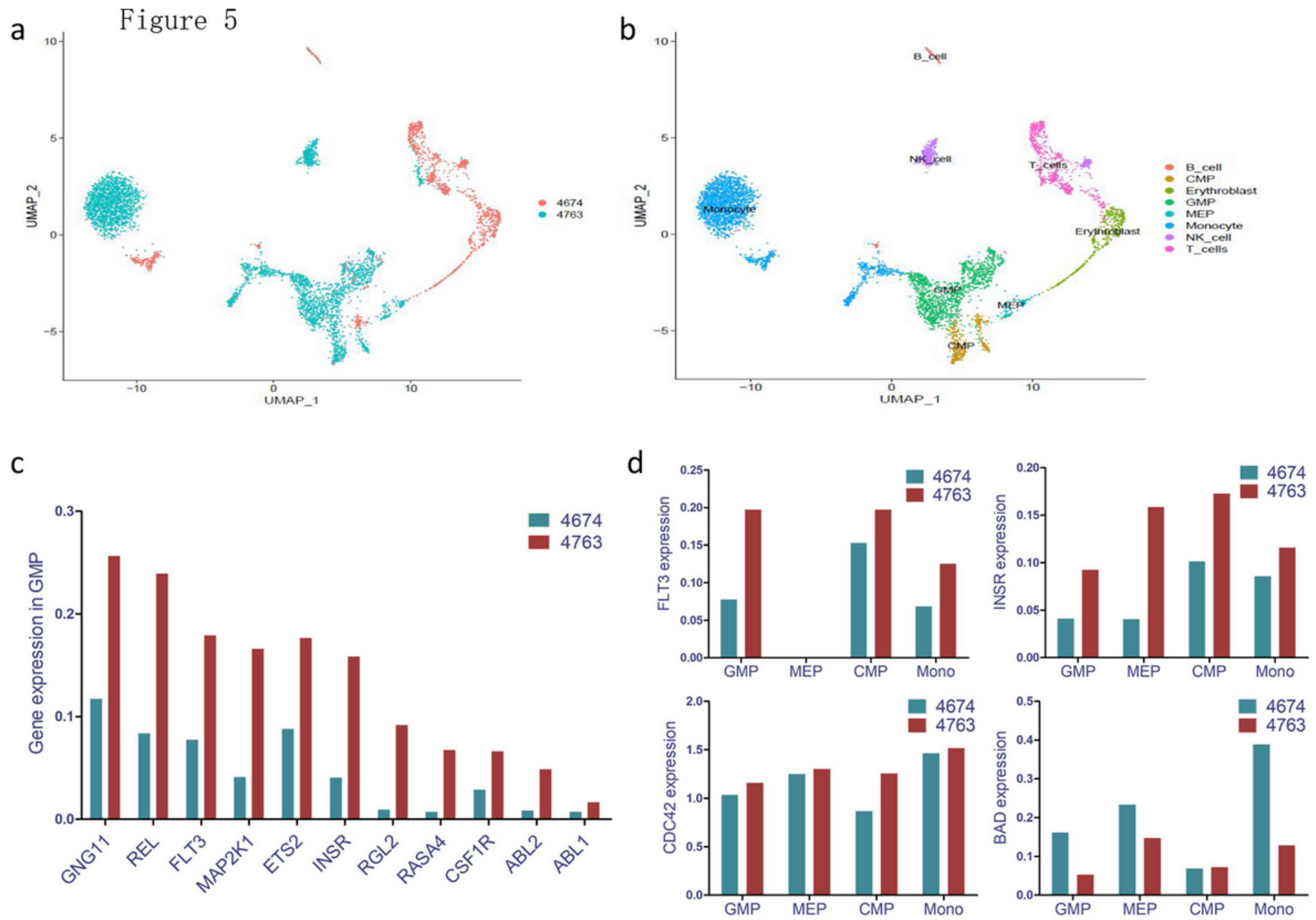

Figure 5

Single-cell RNA transcription group sequencing revealed the linear association of gene mutations with activation of targeted signaling

(a) Patient presented with several abnormal cell groups (blue) after disease progression (orange, 4674 before leukemia transformation and blue, 4763 after leukemia transformation). (b) Cluster analysis suggested that abnormal GMP, CMP, MEP, and monocytes increased in the AML stage. Cells are integrated and shown in two samples. Cells type are recognized and signed in different colors. (c) Integrated analysis indicated that several RAS signaling related-genes were highly expressed in GMP after disease progression. (d) RAS signaling related-genes, such as FLT3, INSR, and CDC42 were also highly expressed in MEP, CMP, and Mono groups. However, apoptosis-related gene BAD is down-regulated in GMP, MEP, and CMP. 